\title{
An automatic segmentation and classification framework for anti-nuclear antibody images
}

\author{
Chung-Chuan Cheng ${ }^{1}$, Tsu-Yi Hsieh², Jin-Shiuh Taur ${ }^{1 *}$, Yung-Fu Chen ${ }^{3,4,5^{*}}$ \\ From 35th Annual International Conference of the IEEE Engineering in Medicine and Biology Society: Work- \\ shop on Current Challenging Image Analysis and Information Processing in Life Sciences \\ Osaka, Japan. 3-7 July 2013
}

\author{
* Correspondence: jstaur@nchu. \\ edu.tw; yfchen@ctust.edu.tw \\ ${ }^{1}$ Department of Electrical \\ Engineering, National Chung Hsing \\ University, Taichung, Republic of \\ China: Taiwan \\ ${ }^{3}$ Department of Healthcare \\ Administration, Central Taiwan \\ University of Science and \\ Technology, Taichung, Republic of \\ China: Taiwan
}

\begin{abstract}
Autoimmune disease is a disorder of immune system due to the over-reaction of lymphocytes against one's own body tissues. Anti-Nuclear Antibody (ANA) is an autoantibody produced by the immune system directed against the self body tissues or cells, which plays an important role in the diagnosis of autoimmune diseases. Indirect ImmunoFluorescence (IIF) method with HEp-2 cells provides the major screening method to detect ANA for the diagnosis of autoimmune diseases. Fluorescence patterns at present are usually examined laboriously by experienced physicians through manually inspecting the slides with the help of a microscope, which usually suffers from inter-observer variability that limits its reproducibility. Previous researches only provided simple segmentation methods and criterions for cell segmentation and recognition, but a fully automatic framework for the segmentation and recognition of HEp-2 cells had never been reported before. This study proposes a method based on the watershed algorithm to automatically detect the HEp-2 cells with different patterns. The experimental results show that the segmentation performance of the proposed method is satisfactory when evaluated with percent volume overlap (PVO: 89\%). The classification performance using a SVM classifier designed based on the features calculated from the segmented cells achieves an average accuracy of $96.90 \%$, which outperforms other methods presented in previous studies. The proposed method can be used to develop a computer-aided system to assist the physicians in the diagnosis of auto-immune diseases.
\end{abstract}

\section{Introduction}

The immune system enables us to resist infections by counteracting invading organisms. Autoimmune disease is a disorder of immune system due to over-reaction of lymphocytes against one's own body tissues [1]. Common autoimmune diseases include Hashimoto's thyroiditis, rheumatoid arthritis, diabetes mellitus type 1 , and lupus erythematosus. Anti-Nuclear Antibody (ANA) is an autoantibody produced by the immune system directed against the self body tissues or cells. The ANA test widely used to detect antibody in the blood plays an important role in the diagnosis of autoimmune diseases. When a particular antibody pattern has been detected, the patient may have the possibility of acquiring certain autoimmune diseases.

(c) 2013 Cheng et al.: licensee BioMed Central Ltd. This is an Open Access article distributed under the terms of the Creative Commons Attribution License (http://creativecommons.org/licenses/by/2.0), which permits unrestricted use, distribution, and reproduction in any medium, provided the original work is properly cited. The Creative Commons Public Domain Dedication waiver (http:// creativecommons.org/publicdomain/zero/1.0/) applies to the data made available in this article, unless otherwise stated. 
Indirect ImmunoFluorescence (IIF) technique applied on HEp-2 cell substrates provides the major screening method to detect ANA patterns in the diagnosis of autoimmune diseases. It produces the ANA images with distinct fluorescence intensities and staining patterns through IIF slides. Currently, the ANA patterns are inspected by experienced physicians to identify abnormal cell patterns, which is a laborious task and may cause harm to physicians' eyes. It is not easy to train a qualified physician in a short term. Furthermore, manual inspection suffers from the difficulties, such as intraand inter-observer variability, that limit the reproducibility of IIF readings [2-5].

Although previous studies have proposed several methods for automatic segmentation of ANA cells [6,7] and criteria for recognition of cell patterns [3,6,8-10], a fully automatic segmentation and recognition framework has never been developed so far. In this study, we propose a framework based on the watershed approaches to automatically segment the HEp-2 cells. It is a crucial preprocessing step for a computer aided system to classify the cell patterns to provide information to assist physicians in disease diagnosis and treatment.

Since the cytoplasm of HEp-2 cells is invisible in the IIF images, in what follows, the term "cell" means cell nucleus, "foreground" indicates the cell region, and "background" denotes the rest of the image. The rest of this paper is organized as follows. Section "Related Works" reviews the techniques used for ANA image segmentation and cell recognition in previous studies. Section "Segmentation of ANA Cells" describes the methods proposed in this study for the segmentation of ANA cells. Classification of ANA cell patterns is demonstrated in section "Cell Classification of ANA Images". Finally, discussions, conclusions, and future works are made in sections "Discussion" and "Conclusion and Future Work".

\section{Related works}

In this section, the methods proposed in previous investigations for the segmentation and classification of ANA cell images are presented.

\section{ANA image segmentation}

Perner et al. [6] used image processing techniques, including image transformation, histogram equalization, Otsu thresholding [11], and morphological operation, to obtain a binary mask for segmenting the cells from the ANA images. By modifying the methods, Huang et al. [7] presented two adaptive automatic segmentation frameworks to precisely extract the ANA cells. In their studies, the first framework classified an image into two categories, i.e., sparse and mass cell regions, based on the number of connected regions. Depending on the category of the images, different color spaces and processing techniques were adopted for cell segmentation. Morphological operations were also used to obtain smooth segmentation results. It was demonstrated to be able to deal with the segmentation of different patterns of IIF images. On the other hand, in the second framework, watershed segmentation [12] was applied on the green channel of the RGB images, followed by region merging and elimination to obtain the cell boundaries. If the number of regions in the obtained image was larger than a pre-defined threshold, the framework converted the original image into CMY color space and performed markercontrolled watershed segmentation [13] on the cyan color component. It was reported that the segmentation performance achieved an overall sensitivity of $94.7 \%$. 
Creemers et al. [14] proposed a unsupervised segmentation algorithm, based on iterative global Otsu thresholding and morphological opening operation, to support IIF testing. It was reported to have the capability to split connected regions into individual regions with an average accuracy of $89.57 \%$.

\section{ANA cell recognition}

Perner [8] presented the first study on fluorescent image analysis, feature extraction and classification. Then, an automatic cell recognition approach based on a variety of features, including size, color density, and number of cells, extracted from the segmented images was proposed [6]. For the cells with identical color density, features including standard deviation, mean shape factor, mean of perimeter, and standard deviation of perimeter, were further extracted. Data mining techniques, including Boolean model and decision tree induction, were then used to label the cell regions. Finally, human experts tagged each labeled region with a semantic label. Based on the aforementioned methods, Sack et al. [3] presented a system to automatic classify HEp-2 fluorescent patterns with a classification accuracy greater than $83 \%$.

According to the fluorescence intensity, Soda and Iannello [9] classified the ANA images into a variety of patterns. They further proposed a framework consisting of hybrid rule-based multi-expert systems for the classification of ANA patterns with an overall error rate of $2.7-5.8 \%$ [15]. The framework extracted the features including the first, second, and fourth moments of the gray-level co-occurrence matrix, Zernike moments, as well as the coefficients of discrete cosine transform (DCT) and discrete wavelet transform (DWT). Based on the efforts of previous researches, Rigon et al. [16] proposed a comprehensive system based on two approaches, in which the first approach discriminated the positive cells from the negative and weakly positive cells based on the features of fluorescence intensity, whereas the second one recognized the staining pattern of the positive cells. The performance of positive/negative recognition ranges from $87 \%$ to more than $94 \%$, whereas the staining pattern classification accuracy of the main classes, i.e. homogeneous cells, peripheral nuclear cells, speckled cells, nucleolar cells, and artefacts, ranges from $71 \%$ to $74 \%$.

Elbischger et al. [17] developed an iterative thresholding algorithm for processing HEp- 2 cells and a cell classifier for detecting auto-immune diseases. Features including area to perimeter ratio, variance, 30th and 60th normalized percentiles, percentile range, dent number, auto-covariance percentage, and roundness, were extracted from the segmented cells and used for cell classification. The system was reported to be capable of distinguishing 5 different patterns with an overall accuracy of $93 \%$ based on the dataset consisting of 982 ROIs extracted from 38 images.

Recently, Huang et al. [18] employed the self-organizing map (SOM) to identify the fluorescence patterns of HEp-2 cells. Fourteen features, including the perimeter, area, and histogram uniformity of the cell; area and average intensity of the inside and perimeter areas of the cell; higher and lower intensity ratios of the inside area, perimeter area, and whole area of the cell; and standard deviation of the inside area of the cell, were used for designing the classifier with an average accuracy of 92.4\%. In [19], the EUROPattern designed based on k-nearest neighbor algorithm, was compared with the conventional visual IIF evaluation with the sensitivity and specificity achieving $100 \%$ and $97.5 \%$, respectively. In addition, it was shown that $94.0 \%$ of all the main antibody 
patterns, including the positive patterns, i.e., homogenous, speckled, nucleolar, centromere, nuclear dotted, and cytoplasmic patterns, as well as the negative patterns, could be correctly recognized.

\section{Segmentation of ANA cells}

As recommended by Center for Disease Control (CDC) [20,21], in this study, the IIF slides were prepared at 1:80 serum dilution, and the ANA images were acquired by a digital camera mounted on a fluorescence microscope at a 40 -fold zoom. The images were stored with the format of 24-bit RGB color depth and a resolution of $3136 \times 2352$ pixels. As shown in Figure 1, the ANA cells are classified into six categories: diffused, peripheral, nucleolar, coarse-speckled, fine-speckled, and discrete-speckled patterns. A dataset, consisting of 196 images classified into 37 diffused, 29 peripheral, 5 nucleolar, 94 coarse-speckled, 1 fine-speckled, and 30 discrete-speckled images by an expert (Dr. Hsieh), was used for the experiments. The procedure of the proposed method is illustrated in Figure 2.

Since the original images are stained by green dye, the proposed method extracts only the green channel from the original RGB ANA images for processing. In order to reduce the computation time, the images are downsized from $3136 \times 2352$ to $1024 \times 768$ pixels. It was found that images at this resolution can still provide enough information for the segmentation and classification of cell patterns. Figure 3 shows an example of ANA image and its corresponding green-channel. As described in the following 3 subsections, the proposed segmentation method divided into 3 procedures, i.e. pre-classification, cell detection 1, and cell detection 2, is presented. The parameters used for these 3 procedures are described in subsection "Parameters for cell segmentation". Finally, the segmentation results are demonstrated in subsection "Segmentation results".

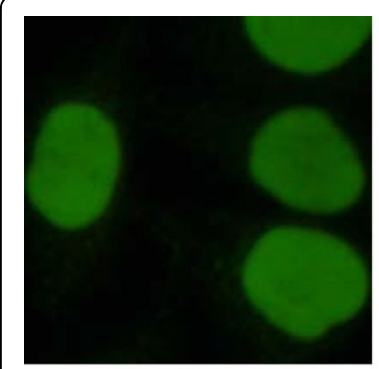

(a)

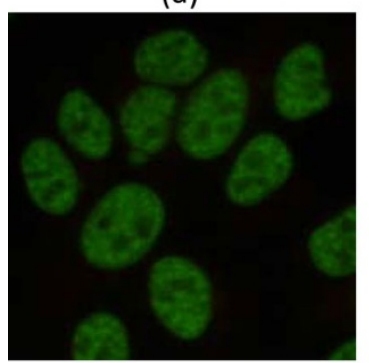

(d)

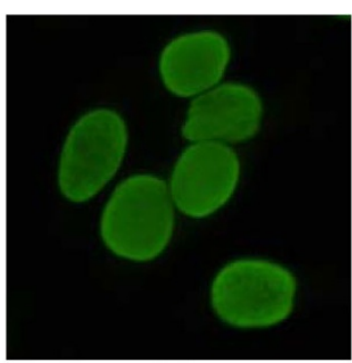

(b)

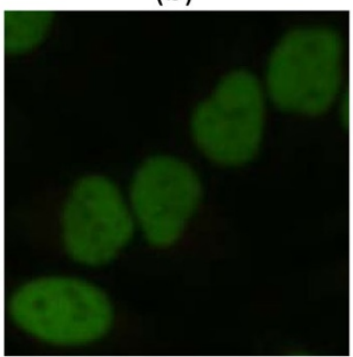

(e)

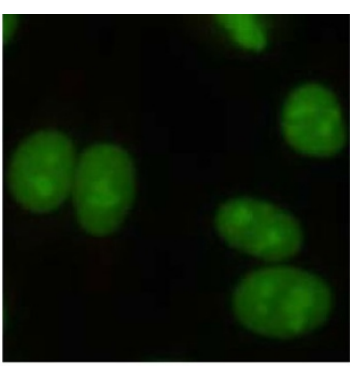

(c)

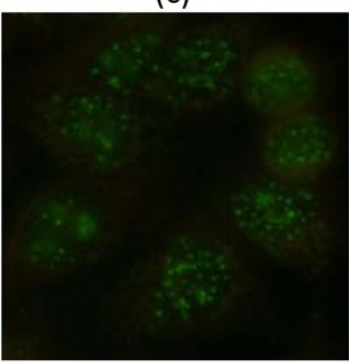

(f)

Figure 1 Example of ANA images. Images classified into (a) diffused, (b) peripheral, (c) nucleolar, (d) coarse-speckled, (e) fine-speckled, and ( $f$ ) discrete-speckled patterns. 




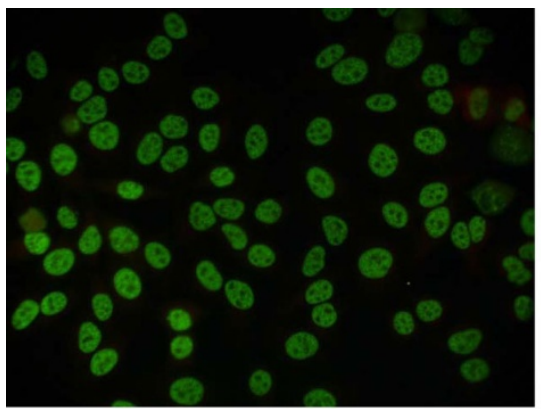

(a)

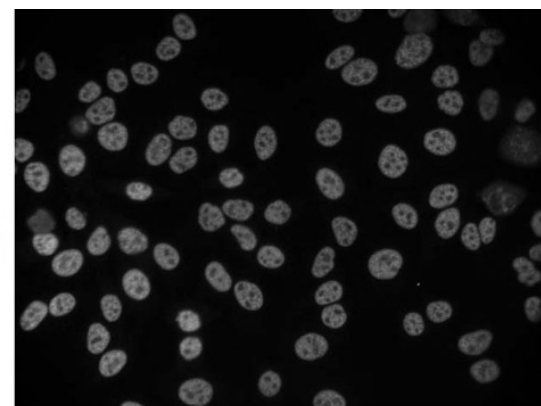

(b)

Figure 3 Green-channel image. Example of (a) an original ANA image and (b) its green-channel image.

\section{Pre-classification}

Automatic segmentation of ANA images cannot be handled in a unified way because the characteristics of the images in different categories are quite dissimilar. For example, the discrete speckled cells look like irregular broken blobs and are significantly different from the cells of other 5 categories that appear as elliptic blobs but still having diverse appearances (cf. Figure 1). Thus, the images are pre-classified according to their differences in image patterns before conducting cell segmentation. In the pre-classification stage, the images are divided into two groups. Images with larger grey-level variance or more regions contained in the foreground are assigned to the first group, and the rest of the images are assigned to the second group. The images in these two groups are segmented using different methods as detailed in subsections "Cell detection 1" and "Cell detection $2^{\prime \prime}$. The procedure of pre-classification is summarized as follows:

1) First, Otsu thresholding algorithm is used to roughly separate the foreground regions from the background.

2) The closing morphological operation is employed to fill the holes and to eliminate small regions in the foreground.

3) If the number of foreground regions in an image is larger than the threshold, th_num, or its foreground regions contain staining noises with variance higher than the threshold, $\boldsymbol{t h} \_\boldsymbol{f g}_{-} \boldsymbol{v a r}$, it is segmented using "Cell detection 1"; otherwise "Cell 
detection 2" is adopted. In this study, the thresholds $\boldsymbol{t h} \_\boldsymbol{n} \boldsymbol{u m}$ and $\boldsymbol{t h} \boldsymbol{f} \boldsymbol{g} \_\boldsymbol{v a r}$ are set to 200 and 1000 , respectively.

4) For images segmented with "Cell detection 2", the staining noise in the background regions are removed according to their noise level, as defined in the following equation:

$$
\sum_{i=0}^{128}[p(i)>0],
$$

where $i$ indicates the gray level of the image, and $p(i)$ denotes the frequency of gray level $i$ in the image. The threshold of noise level, th_noise, is set to 10 .

\section{Cell detection 1}

The approach is designed for cell detection of the images containing more foreground regions or the gray level in the foreground regions presenting great variance. It consists of two stages: image segmentation and cell extraction. Cells are extracted according to the cell contours obtained from the general watershed segmentation [12] and markercontrolled watershed segmentation [13]. As described below, the procedure of this approach is divided into four steps. Figure 4 illustrates the results obtained from individual steps.

1) The histogram equalization is applied to the original image in Figure 4(a), and then the pixels with gray level greater than 240 are considered as the initial markers, as presented in Figure 4(b).

2) As demonstrated in Figure 4(c), the original image is smoothed by the morphological opening operation using a disk-shaped structuring element with a radius of 15 .

3) The Difference between the original image and the smoothed image is obtained, Figure 4(d), and is converted into a binary image, Figure 4(e), by applying the Otsu thresholding method.

4) The initial markers are superimposed on the thresholded image shown in Figure 4 (e), followed by applying the same opening morphological operation mentioned in the $2^{\text {nd }}$ step to obtain the marker image, Figure 4(f), used for marker-controlled watershed segmentation. The flowchart of marker extraction is depicted in Figure 5.

As described in Steps 5-7, 3 types of watershed images are obtained based on the original image, smooth image, and marker image, and are used for cell segmentation.

5) The original image shown in Figure 4(a) is complemented by subtracting each pixel value from 255 for conducting watershed segmentation. Figure 6(a) shows the background watershed image (b-ws) superimposed on the original image.

6) Furthermore, Gaussian differentiation parameter with $\sigma=2$ and thresholding parameter of $\boldsymbol{h}$-minima suppression with $\boldsymbol{t} \boldsymbol{h} \_\boldsymbol{h} \mathbf{1}=0.12$ [22] are applied on the smoothed image for conducting watershed segmentation to obtain the foreground watershed segmentation image (f-ws). Figure 6(b) presents the "f-ws" image superimposed on the original image.

7) Similar to the foreground watershed segmentation, the smoothed image is first filtered by the Gaussian differentiation and minima suppressed by $h$-minima transform, which is then superimposed by the marker image and the "b-ws" image to obtain the foreground marker-controlled watershed (fmc-ws) image used for marker-controlled watershed segmentation. Figure 6(c) shows the foreground marker-controlled watershed (fmc-ws) image. 


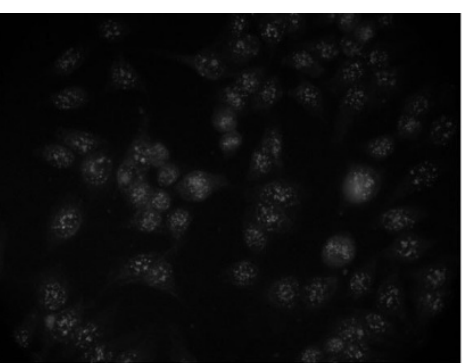

(a)

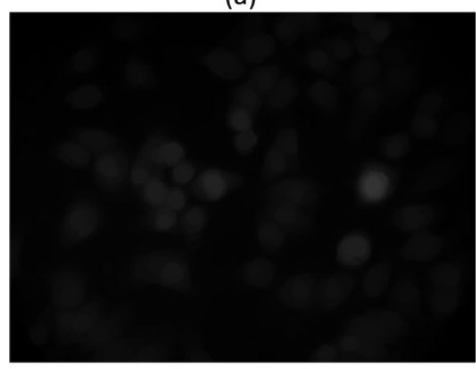

(c)

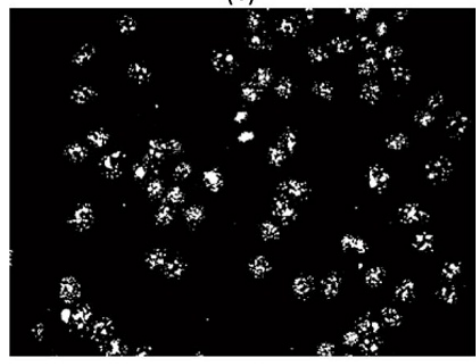

(e)

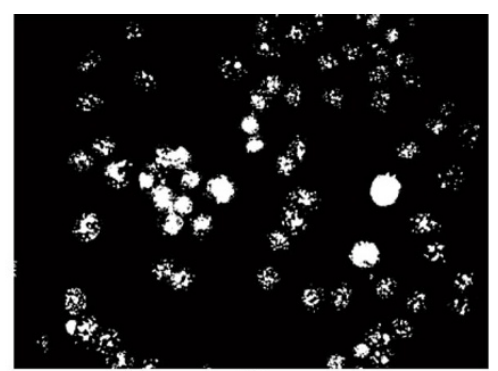

(b)

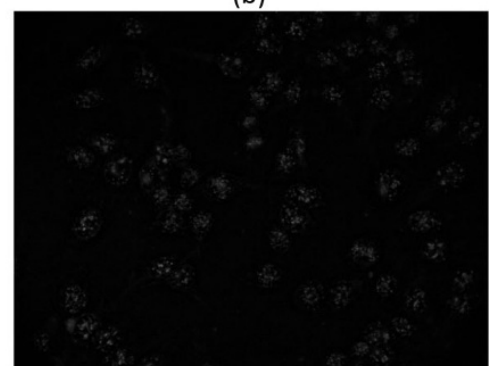

(d)

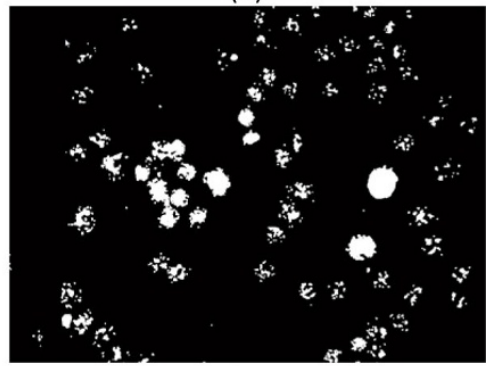

(f)

Figure 4 Extraction of marker images. (a) The original, (b) initial marker, and (c) smooth images. (d) Difference image obtained from the original image and the smooth image. (e) Image after performing Otsu thresholding on difference image and (f) the marker image after applying opening morphological operation.

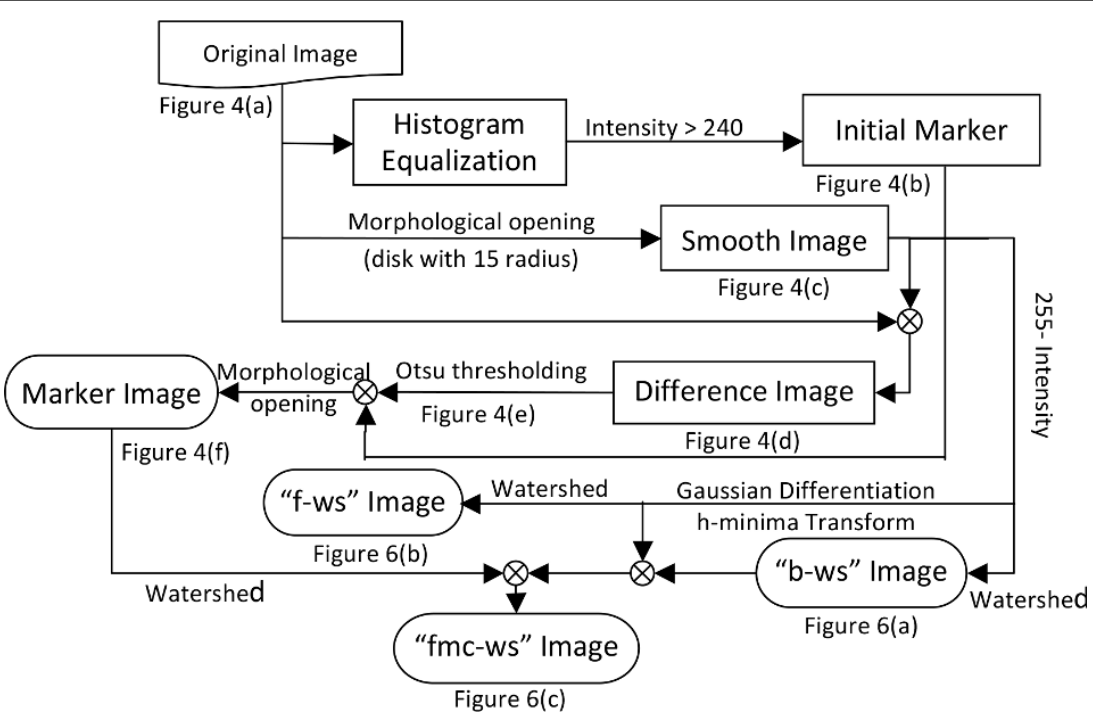

Figure 5 Flowchart of marker extraction. 


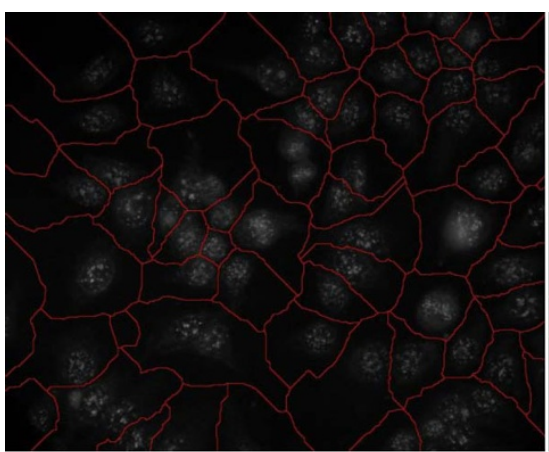

(a)

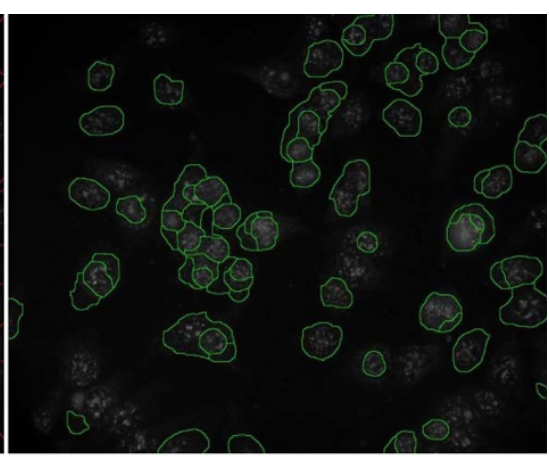

(b)

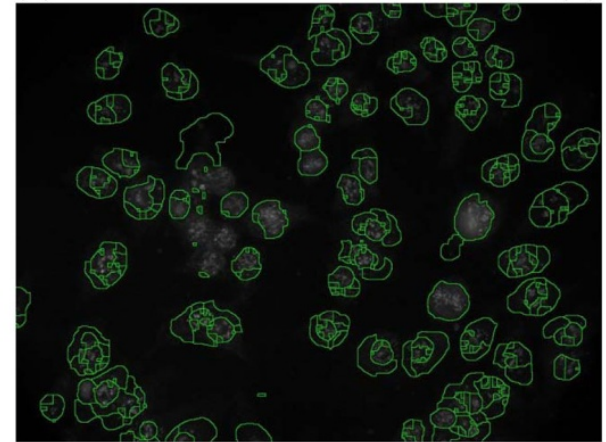

(c)

Figure 6 Examples of three types of watershed images. (a) Background-watershed (b-ws) image, (b) foreground-watershed (f-ws) image, and (c) foreground marker-controlled-watershed (fmc-ws) image superimposed on the original image.

These 3 types of watershed images were further used for cell segmentation. As demonstrated in Figure 6, it can be observed that the "b-ws" image is effective in splitting cells that are close to each other. The blobs in "fmc-ws" are mostly oversegmented with unsmooth contours, resulting in a failure to effectively delineate the cell contours. On the other hand, the "f-ws" image is unable to detect some of the cell regions. Consequently, in the cell extraction stage of "Cell detection 1", the three types of watershed images and the marker image are combined to precisely extract cell boundaries.

As illustrated in Figure 7, the strategies for cell extraction using the watershed images are described in the following steps:

1) The three watershed images, i.e., "b-ws", "f-ws" and "fmc-ws", are all binary images. The cell contours in "f-ws" and "fmc-ws" images are labeled as ZERO, otherwise ONE, followed by the removal of background regions to obtain the watershed mask images shown in Figure 8(a) and 8(b), respectively.

2) The cell regions are extracted from the "fmc-ws" image according to the perimeters of the connected regions, since it can potentially detect more cell regions than "f-ws". The regions whose areas larger than the threshold $\boldsymbol{t h}$ _area are justified by "ellipse test" and considered as the cells after having passed the test.

3) For the regions with areas smaller than the threshold th_area, closing morphological operation is conducted to merge smaller regions. The merged regions are then justified by ellipse test for cell extraction. As demonstrated in Figure 9, the small inner regions of the remains are merged to larger regions. 


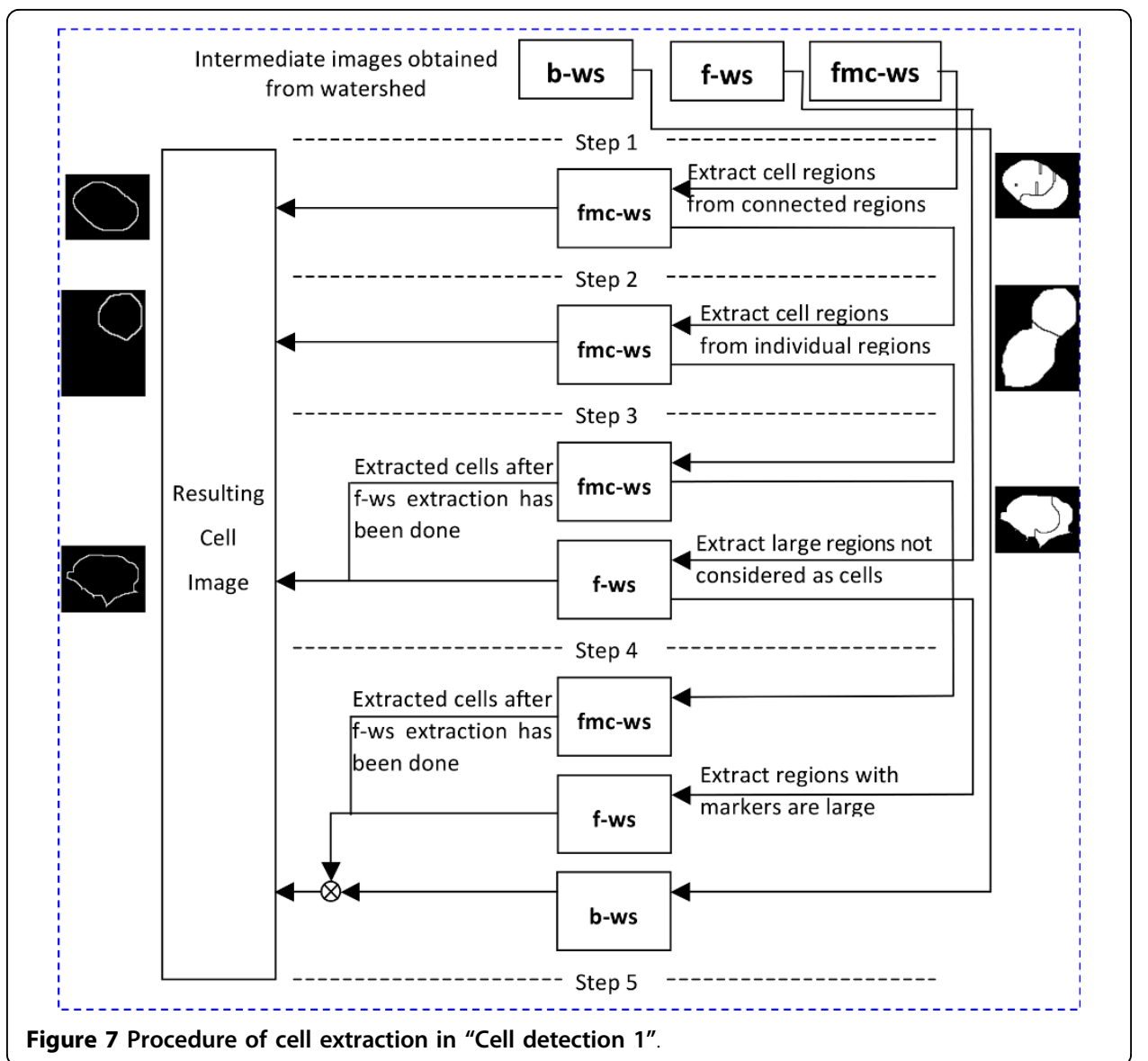

4) For the regions, which are not deemed as ellipses from the remains of the "fmc-ws" at Step 3 and the "f-ws", having areas higher than th_area and containing markers in the corresponding locations, they should be treated as the candidate cells. Due to the fact that the blobs of "f-ws" are more similar to real cells than "fmc-ws", "f-ws" is used to perform cell extractions before applying "fmc-ws" here.

5) Most of the cells in "f-ws" and "fmc-ws" should have been extracted at the previous 4 steps, but some regions may not be detected because their markers are large enough to cover the edges of the regions. Figure 10(a) demonstrates the cells detected at Steps 1-4. However, as shown in Figure 10(b), watershed segmentation may fail when detecting the cells whose corresponding markers are large enough to cover the whole candidate cell. Hence, if the markers existed in the marker-image are larger than the threshold $\boldsymbol{t h}$-area2, the watershed segmentation (with a parameter of $\boldsymbol{h}$-minima transform $\boldsymbol{t h} \boldsymbol{h} \mathbf{h}$ ) is performed in the corresponding region of the smooth image. Here, only the corresponding region of "b-ws", as shown in Figure 10 (c), is considered for extracting the cells.

Due to the fact that the real HEp-2 cells usually appear as ellipses, the cells can be justified by "ellipse test". It is used to justify whether a region contains a cell or not. Given a region $r_{i}$, the error between $r_{i}$ and a real ellipse $r_{i}^{I}$ is defined as:

$$
e_{i}=\frac{\left|r_{i}^{\prime}\right|}{\left|r_{i}\right|} \text {, with } r_{i}^{\prime}=r_{i} \mathrm{XOR} r_{i}^{I} \text { and } r_{i}^{I}=\operatorname{Ellipse}(a, b, \theta)
$$




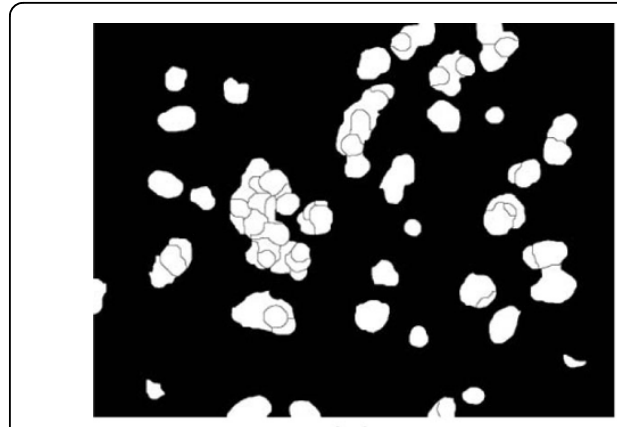

(a)

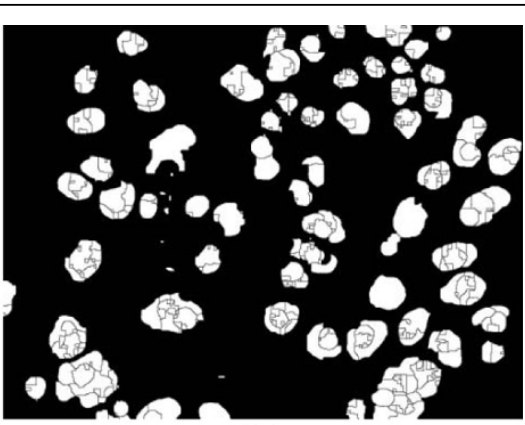

(b)

Figure 8 Mask images of cell regions with background removal. (a) "f-ws" image and (b) "fmc-ws" image.

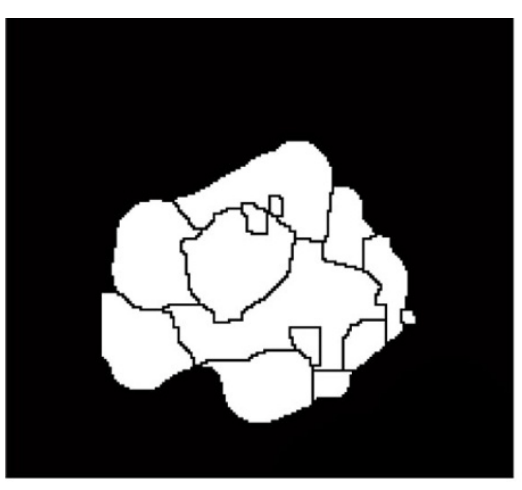

(a)

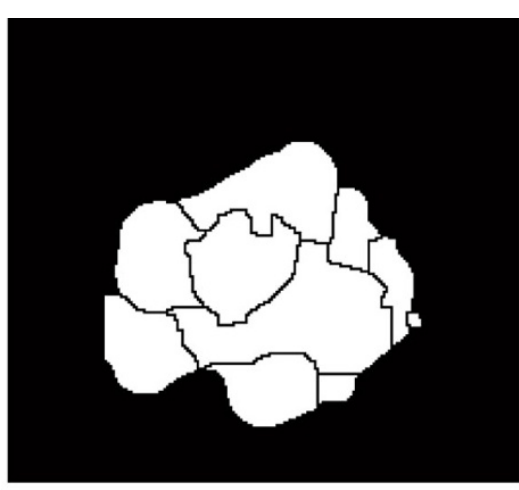

(b)

Figure 9 Merging the small inner regions of a region not justified as a cell. (a) Inner regions which are not justified as cells and (b) larger inner regions obtained by merging smaller inner regions with morphological closing operation.

in which $\left|r_{i}\right|$ denotes the number of pixels in $r_{i}$ and $r_{i}^{I}$ is the estimated ideal ellipse for $r_{i}$, comprising three parameters: major-length $(a)$, minor-length $(b)$, and orientation $(\theta)$. The lengths of major axis and minor axis are both computed according to the centroid of $r_{i}$. If the error function of a region is equal to zero, the region is deemed as an ideal ellipse. Figure 11 depicts a region and its estimated ideal ellipse. If the error of a region is lower than the threshold th_error, it is marked as a cell; otherwise, it may be treated as one of following cases: not a cell, an incomplete cell, or a connected region.

\section{Cell detection 2}

The approach is applied to the images containing less foreground regions $\left(\boldsymbol{t h} \_\boldsymbol{n} \boldsymbol{u m}<200\right)$ and less staining noise $\left(\boldsymbol{t} \boldsymbol{h} \_\boldsymbol{f g} \_\boldsymbol{v a r}<1000\right)$ detected in the foreground. As shown in Figure 12, the procedure is very similar to that in "Cell detection 1", except that the image segmentation uses only the "b-ws" (red) and "f-ws" (green) watershed images without considering the "fmc-ws" image. The procedure is described as follows:

1) Remove the background regions of the "f-ws" image.

2) Extract cell regions from "f-ws".

3) Because of the characteristics of watershed segmentation, the adjacent regions form connected regions. The regions which are not extracted in step 2 may be fake 


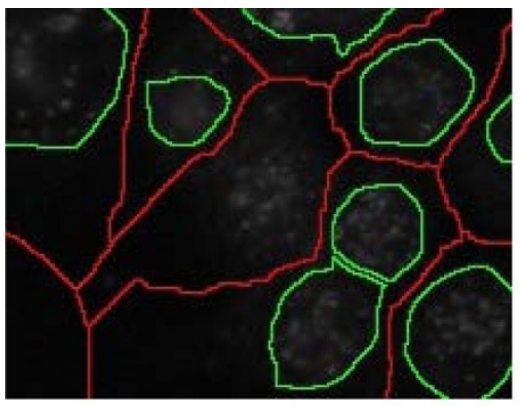

(a)

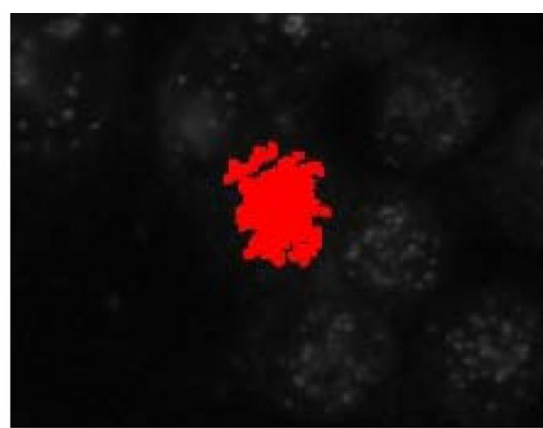

(b)

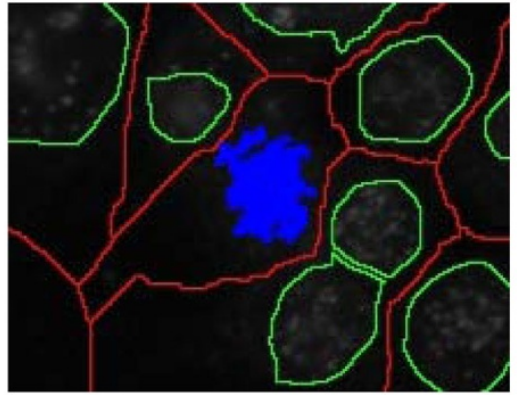

(c)

Figure 10 Extracting cells for regions with large markers. Illustration of (a) 8 detected cells (green) and 1 undetected cell, (b) the marker corresponding to the b-ws region containing the undetected cell, and (c) superimposition of images shown in (a) and (b).

connected regions, which can be split by using the information embedded in the "b-ws" image. As illustrated in Figure 13(a), a sub-region, which connects two watershed regions, with a line in the "b-ws" image crossing it will be eliminated, resulting in the separation of two cell blobs, Figure 13(b). Subsequently, watershed segmentation (with a designated parameter of h-minima transform, $\boldsymbol{t h} \_\boldsymbol{h} \mathbf{1}$ ) is further performed on the

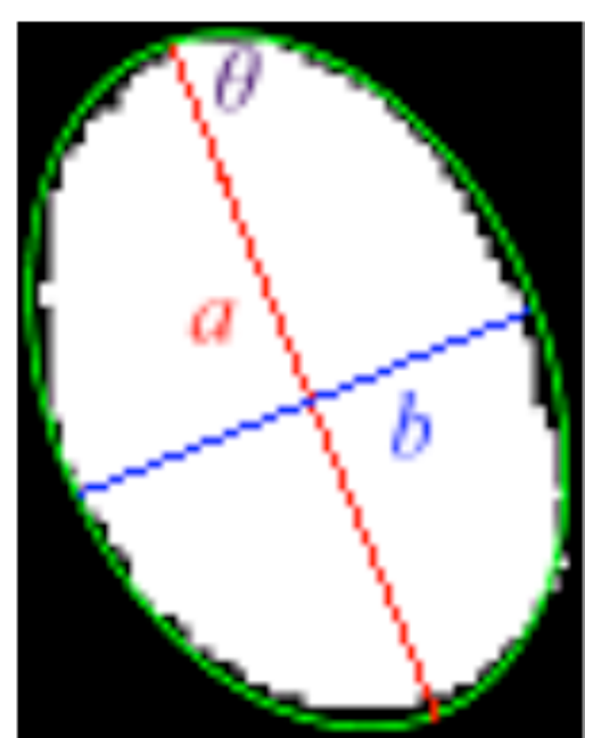

Figure $11 \mathrm{~A}$ region $r_{i}$ and its fitted ellipse $r_{i}^{\prime}($ green). 


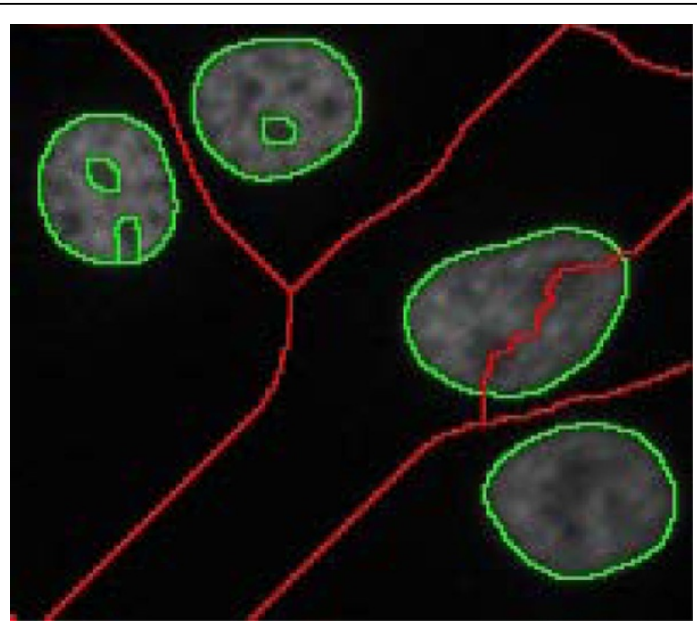

Figure 12 An example of cell segmentation using "Cell Detection 2".

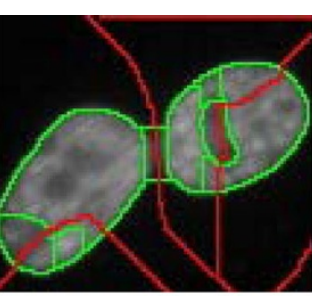

(a)

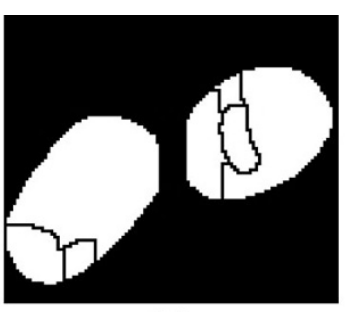

(b)

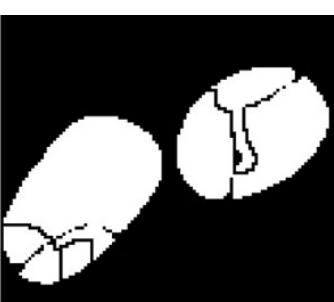

(c)

Figure 13 Splitting of a fake sub-region. (a) A fake sub-region with a line in "b-ws" crossing it is split to (b) two separated candidate cell regions. (c) Subsequent watershed segmentation is performed on the candidate cell regions on "f-ws".

individual cell regions appeared on "f-ws", Figure 13 (c). The sub-regions in the refined cell regions are merged and justified by "ellipse test" afterward.

4) For connected cell regions which can't be split at step 3, all possible combinations of sub-regions will be tested to obtain combinations of sub-regions which are similar to ellipses. Once the best combination has been obtained, the cell regions can be wellseparated from the background. Figure 14 illustrates the procedure in splitting the region containing three candidate cell regions. A connected region $r_{i}$ consisting of $N_{i}$ sub-regions can be indicated as:

$$
r_{i}=\left\{r_{i, 1}, r_{i, 2}, \cdots r_{i, j}, \cdots r_{i, N_{i}}\right\}
$$

The error function of the $k_{\mathrm{th}}$ combination of sub-regions, $c o m b_{k}$, can be calculated according to:

$$
e_{k}=\frac{\left|\operatorname{comb}_{k}^{\prime}\right|}{\left|\operatorname{comb}_{k}\right|}
$$

where $c o m b_{k}^{\prime}=\operatorname{comb}_{k}$ XOR $c o m b_{k}^{I}$ with $c o m b_{k}^{I}$ denoting the estimated ideal ellipse of $c o m b_{k}$. If the combination with smallest error, $k^{\prime}=\arg \min _{k} e_{k}$, has been found, the connected region can be split to isolated regions $r_{i}^{\prime}$ accordingly. As shown in Figure 14(c), "b-ws" image is superimposed on the "f-ws" image to form 17 sub-regions. The 


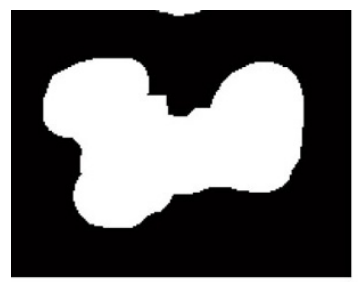

(a)

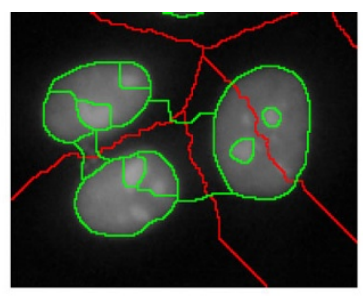

(b)

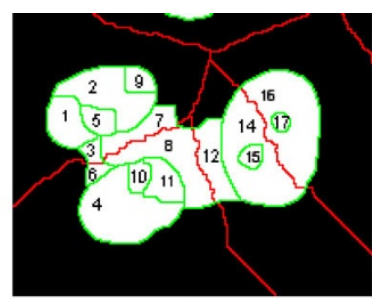

(c)

Figure 14 Example of splitting a connected region. (a) A connected region is split into 3 cell regions by (b) superimposing "b-ws" image on "f-ws" image and then (c) determining combined sub-regions and discarded sub-regions.

combinations with smallest errors include $\left\{r_{i, 1}, r_{i, 2}, r_{i, 5}, r_{i, 9}\right\},\left\{r_{i, 4}, r_{i, 10}, r_{i, 11}\right\},\left\{r_{i, 14}, r_{i, 15}\right.$, $\left.r_{i, 16}, r_{i, 17}\right\}$, and $\left\{r_{i, 3}, r_{i, 6}, r_{i, 7}, r_{i, 8}, r_{i, 12}\right\}$. After the ellipse tests, the sub-region combinations $\{1,2,5,9\},\{4,10,11\}$, and $\{14,15,16,17\}$ are merged into 3 cell regions, while the combination $\{3,6,7,8,12\}$ is discarded. Once a connected region has been split, the new regions are modified by performing watershed segmentation (with a designated parameter of $\boldsymbol{h}$-minima transform, $\boldsymbol{t} \boldsymbol{h} \boldsymbol{h} \boldsymbol{2}$ ) in their locations corresponding to the locations in the "b-ws" image. The sub-regions $\{3,6,7,8,12\}$ are discarded because their intensities and textures are very similar to the background when the local watershed segmentation has been applied.

5) Due to the fact that the foreground of dataset images may contain inhomogeneous gray levels, some regions can not be detected because they are darker than other regions, even though they can be discriminated by human eyes. In order to detect these regions, global Otsu thresholding is again performed on the remaining image after cell extraction. Detected regions with areas greater than $\boldsymbol{t h}$ _area are considered as cells.

\section{Parameters for cell segmentation}

The parameters used for different stages of cell segmentation are listed in Table 1. The parameters $\boldsymbol{t h} \_\boldsymbol{h} \mathbf{1}$ and $\boldsymbol{t} \boldsymbol{h} \_\boldsymbol{h} \mathbf{2}$ are crucial in effectively suppressing noises and local irregularities in the gradient images. Furthermore, the segmentation results are very sensitive to the parameters, even they are only changed slightly; hence they are set case by case for obtaining complete blobs and avoiding over-segmentation. If the values are too small, the blob will be over-segmented and need more time to find $r_{i}^{\prime}$, which is the combination of sub-regions with the smallest error. In contrast, larger values may cause the watershed to reach a boundary outside the blob and cannot converge at the real boundaries. The procedures of setting the parameters $\boldsymbol{t} \boldsymbol{h} \_\boldsymbol{h} \mathbf{1}$ and $\boldsymbol{t} \boldsymbol{h} \_\boldsymbol{h} \mathbf{2}$ are based on the greedy algorithm.

Table 1 Parameters designated for different stages of cell segmentation.

\begin{tabular}{cccccc}
\hline \multicolumn{2}{c}{ Pre-classification } & \multicolumn{2}{c}{ Cell Detection $\mathbf{1}$} & \multicolumn{2}{c}{ Cell Detection 2} \\
\hline Parameter & Value & Parameter & Value & Parameter & Value \\
\hline th_num & 200 & $\boldsymbol{t h}$ h1 & 0.12 & th_h1 & 0.12 \\
\hline th_noise & 10 & th_h2 & 0.28 & th_h2 & 0.28 \\
\hline th_fg_var & 1000 & th_area & 400 & th_area & 400 \\
\hline & th_error & 0.095 & th_error & 0.095 \\
\hline & th_area2 & 32 & & \\
\hline
\end{tabular}


The parameters, th_area and th_error, are used as the criteria for judging whether a blob is a cell or not. Considering an ANA image with a size of $1024 \times 768$ pixels, the minimum cell size is set to 400 pixels, i.e. th_area $=400$, according to the physician's opinion. Figure 15 compares the errors among regions with different shapes. Note that a perfect ellipse has a zero error. Since HEp-2 cells may be squeezed, superimposed, demolished, or deviated from a perfect ellipse, the value of $\boldsymbol{t h}$ _error (0.095) is determined by greedy algorithm with a grid size of 0.005 to select the optimal threshold with best detecting accuracy according to the 3830 ground-truth cell images extracted from 196 images in the dataset. On the other hand, the parameter $\boldsymbol{t h} \_$area 2 is used to find the markers located in non-recognized cell regions. In the cases of nucleolar and discrete-speckled patterns, the markers could be too small to be used for cell detection. Hence, its value is assigned as th_area $2=32$ for mild restriction.

\section{Segmentation results}

Figure 16 demonstrates the segmentation results of ANA images with 6 different patterns. As shown in this figure, the proposed method performs well on almost all the images with different cell patterns; however, the performance on images of diffuse and discrete-speckled patterns is less satisfactory because the cells of diffused pattern contain more closely connected regions than the other types of cells, whereas the cells of discrete-speckled pattern appear to have less obvious boundaries. Figure 17 compares the segmentation results using the proposed method with examples of the groundtruth images. The ground-truth images were delineated by the technicians trained by one of the authors, Dr. Hsieh. Performance of the segmentation results was evaluated

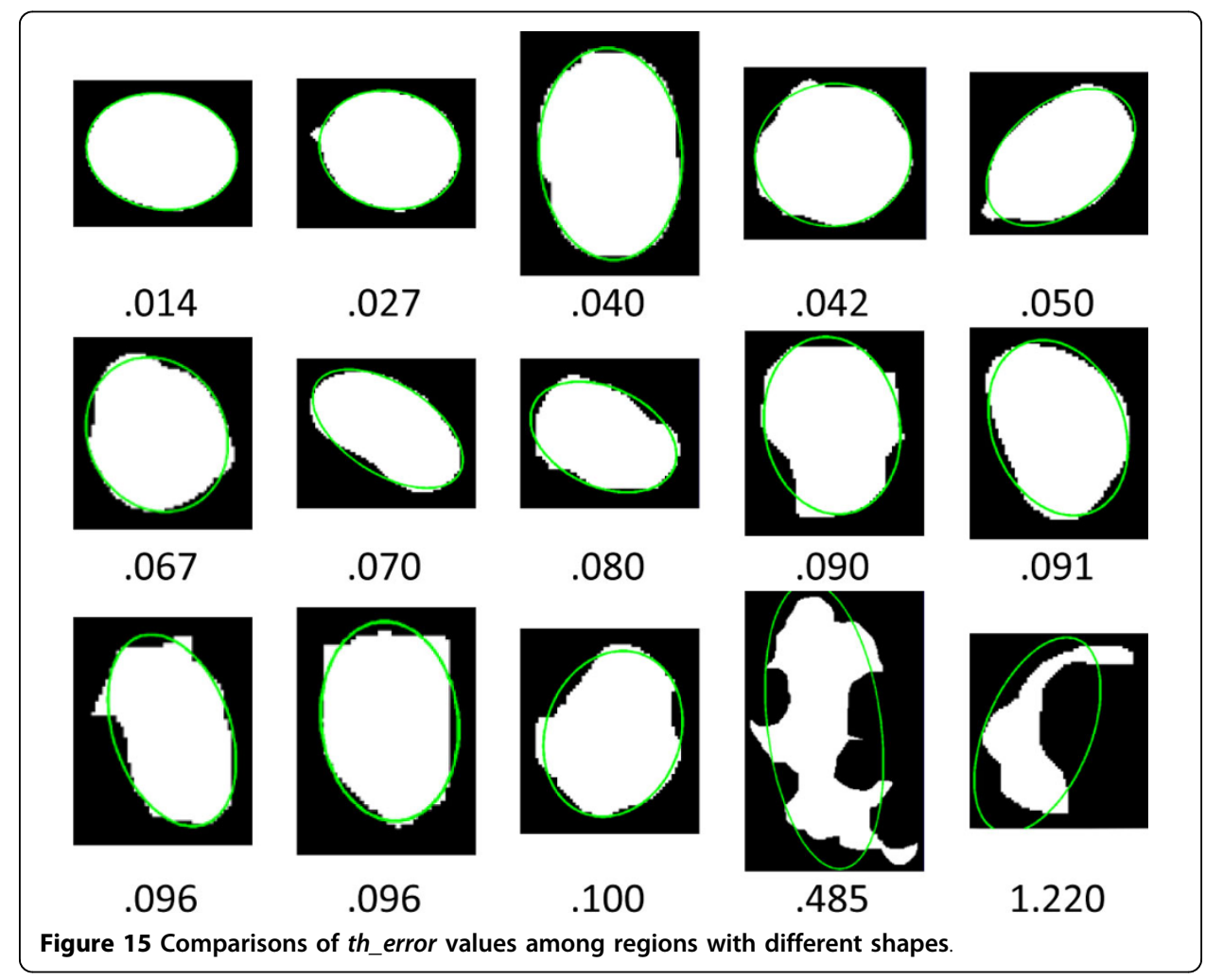




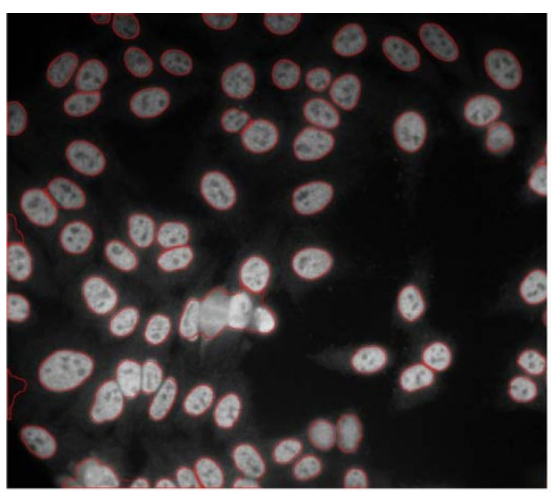

(a)

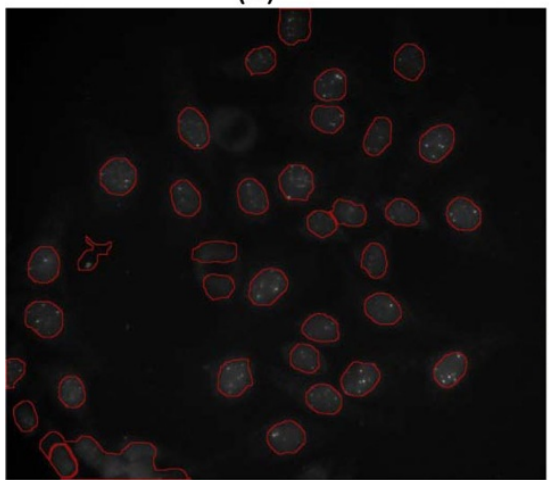

(c)

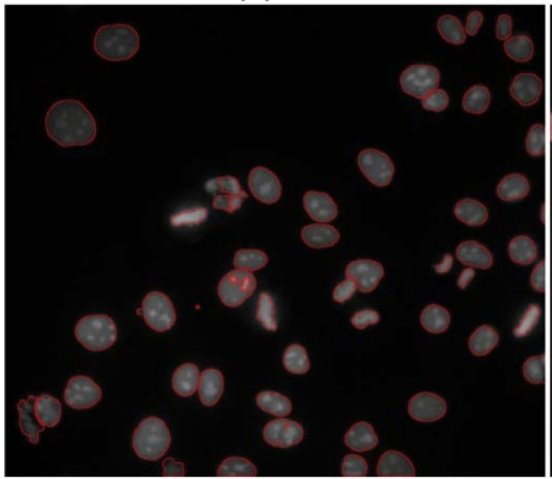

(e)

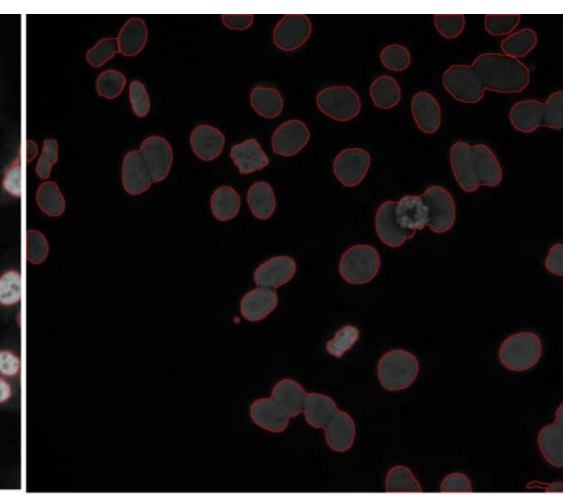

(b)

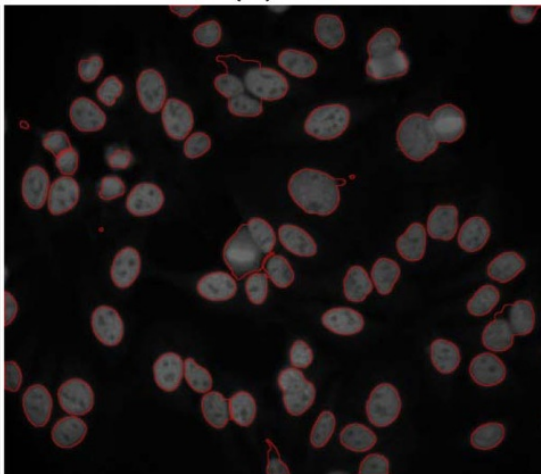

(d)

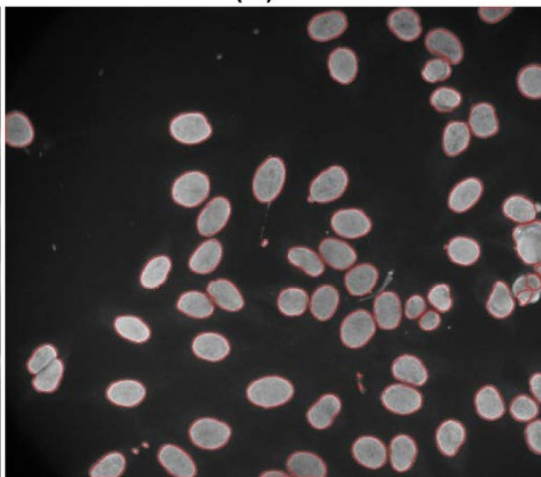

(f)

Figure 16 Examples of segmentation results of ANA images. Segmented cells of images with (a) coarsespeckled, (b) diffused, (c) discrete-speckled, (d) fine-speckled, (e) nucleolar, and (f) peripheral patterns, respectively.

with percent volume overlap (PVO) and percent volume difference (PVD) that had been used widely in previous works [23-26].

Given two contours, denoted by $C_{s}$ and $C_{g}$, obtained from the proposed method and the ground truth, respectively, of the segmented image, PVO and PVD can be calculated from the following formula:

$$
\begin{aligned}
& \operatorname{PVO}\left(C_{s}, C_{g}\right)=\frac{V\left(C_{s} \cap C_{g}\right)}{\left[V\left(C_{s}\right)+V\left(C_{g}\right)\right] / 2} \times 100 \%, \text { and } \\
& \operatorname{PVD}\left(C_{s}, C_{g}\right)=\frac{\left|V\left(C_{s}\right)-V\left(C_{g}\right)\right|}{\left[V\left(C_{s}\right)+V\left(C_{g}\right)\right] / 2} \times 100 \%,
\end{aligned}
$$




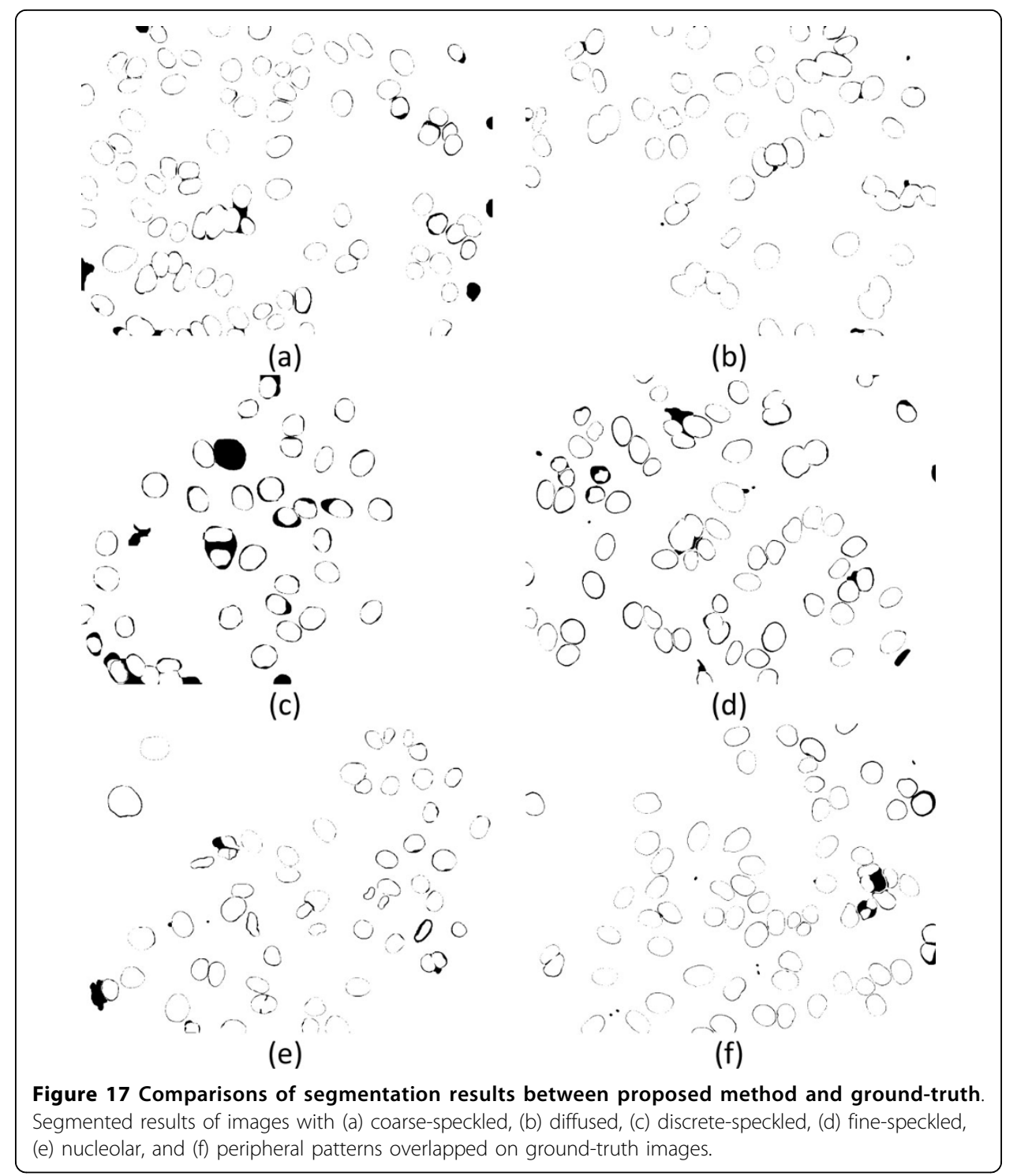

where $V(C)$ indicates the volume of a contour. Table 2 presents the average performance of the segmentation results evaluated based on PVO and PVD. The results show that the proposed method can detect cells accurately in most image cases with the PVO greater than $89 \%$ and the PVD less than $22 \%$. Even for the most difficult

Table 2 Comparisons of cell segmentation performance

\begin{tabular}{cccc}
\hline Pattern & Cell Number & PVO (\%) & PVD (\%) \\
\hline Coarse speckled & 260 & 92.35 & 15.31 \\
\hline Diffuse & 157 & 87.89 & 24.23 \\
\hline Discrete speckled & 195 & 78.03 & 43.95 \\
\hline Fine speckled & 67 & 91.27 & 17.46 \\
\hline Nucleolar & 175 & 91.94 & 16.12 \\
\hline Peripheral & 153 & 93.47 & 13.06 \\
\hline
\end{tabular}

Performance evaluated based on average percent volume overlap (PVO) and percent volume difference (PVD). 
cases appear in cells with discrete-speckled pattern, PVO can still achieve a value over $75 \%$. As a matter of fact, it is not necessary to segment HEp-2 cells with great accuracy. However, the segmentation results must be good enough to extract features to support accurate cell classification, as described in next section.

\section{Cell classification of ANA images}

By considering the effect of astigmatism, the texture details of the cells which are not located at the central field may be lost due to optical aberration. Hence, only the cells located in the central field, accounting to $50 \%$ of the area from the image center, are used for cell classification. A total of 3830 cells extracted from 196 images are classified into 6 different patterns, i.e. diffused (599), peripheral (529), nucleolar (94), coarse-speckled (1956), fine-speckled (56), and discrete-speckled (596), by an experienced physician, Dr. Hsieh. The classified cell patterns are adopted as the ground truth to verify classification performance of the proposed method.

\section{Features for cell classification}

For the purpose of finding suitable features to represent the patterns of ANA images, conventional and the state-of-the-art features are investigated. The conventional features used to describe patterns include statistics of intensity and texture of blobs. The statistics of blob intensity include mean, variance, skewness, and entropy. Tamura features, including coarseness, contrast, and directionality, as well as the Haralick features, including contrast, correlation, energy, and homogeneity, obtained from co-occurrence matrix (GLCM) with $0,45,90$, and 135 degrees, are also used to characterize the blobs. Furthermore, the most frequently used state-of-the-art features, such as fuzzy texture spectrum (FTS) $[27,28]$ and local binary pattern (LBP) [29-31], are also adopted for cell classification in this study.

In addition, by observing the ANA images, a novel feature has been proposed to describe the appearance of blobs from the intensity images. As illustrated in Figure 18, the features obtained from the perimeters and the central areas of the blobs are different between two different cell patterns, such as the peripheral and nucleolar patterns.

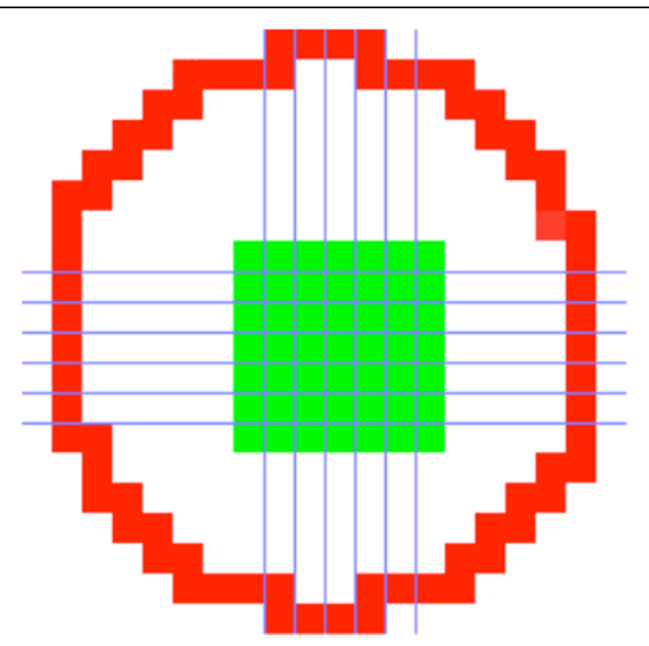

Figure 18 Intensity difference between perimeter (red) and central area (green) 
It can be distinguished by calculating the intensity difference between the perimeter and the central area of a blob according to the following equation:

$$
d_{p c}=P_{\text {avg }}-C_{\text {avg }}
$$

where $P_{\text {avg }}$ denotes the average intensity of pixels located at the perimeter of a blob, and $C_{\text {avg }}$ indicates the average intensity of the central area with a size of $7 \times 7$ pixels.

By observing images in Figure 1, it can be found that different cell patterns contain a variety of regions with different sizes and patterns. For example, although nucleolar and discrete-speckled patterns both contain light regions, the number of light regions in the cells with discrete-speckled pattern is greater than the nucleolar pattern. In contrast, some dark regions can be observed in the coarse-speckled and fine-speckled patterns. These are important and useful characteristics to reduce false cases in discriminating cells with different patterns. A total of 6 features derived from statistics of light and dark regions inside the blobs, including numbers of dark and light regions as well as mean and variance of intensity of dark and light regions, are obtained for cell discrimination.

In total, 129 candidate features were used to represent the patterns of individual ANA images. As indicated in Table 3, the features were grouped into 11 categories, i.e. STATS (3 features), TAMURA (3 features), HARALICK (16 features), FTS (45 features), LR (3 features), DR (3 features), LBP8 (10 features), LBP16 (18 features), LBP24 (26 features), ENTROPY (1 feature) and DPC (1 feature).

\section{Design and validation of cell classifier}

Support vector machine (SVM) is a supervised learning method widely used for classification of data patterns $[32,33]$. A special property of SVM is that it can simultaneously minimize the empirical classification error and maximize the geometric margin of a classifier. It is a powerful methodology for solving problems in nonlinear classification, function estimation, and density estimation, leading to many applications [34].

In this study, SVM classifier was implemented by the LIBSVM tool [35] which supports multi-class classification. Radial basis function (RBF) was selected as the kernel because of its advantages in mapping samples into a higher dimensional space so that

Table 3 Categories of features used for cell classification.

\begin{tabular}{ccc}
\hline Category & $\begin{array}{c}\text { Features } \\
\text { No. }\end{array}$ & Description \\
\hline STATS & 3 & Intensity statistics of blobs: mean, variation, and skewness \\
\hline TAMURA & 3 & Coarseness, contrast, and directionality \\
\hline HARALICK & 16 & Contrast, correlation, energy, and homogeneity in co-occurrence matrix with degrees \\
$0,45,90$, and 135.
\end{tabular}


it can handle the case when the relation between class labels and attributes is nonlinear [36]. The optimal combination of penalty parameters, $C$ and $\gamma$ of the RBF kernel, were determined by dividing the range $2^{-10}-2^{+10}$ into 21 steps, resulting in a total of 441 combinations.

Two experiments for the verification of classification performance of the SVM classifier were conducted: cross validation (CV) and independent training and testing (ITT). For the CV experiment, 5-fold cross-validation was conducted to obtain the optimal parameters $C$ and $\gamma$ in the training phase. On the other hand, the images dataset was randomly divided into training set and testing set, each containing $50 \%$ of the randomly selected images, for ITT. Again in the training phase, 5 -fold cross-validation was used to obtain the optimal combination of parameters $C$ and $\gamma$ based on the training set. The ITT experiment were repeated for 10 times.

Table 4 reveals the resulting accuracy obtained from the CV and 10 ITTs with all of features presented in Table 3. It mimics that the proposed segmentation method is good enough to detect cell contours for extracting features to design a classifier with satisfactory classification accuracy. Additionally, one of the objectives of this study is to select salient features to represent cell patterns.

The sequential backward selection (SBS) [37] was frequently used in many researches for feature selection. In this study, the SVM-RFE (recursive feature elimination) reported to be effective for multi-cluster classification [38], was adopted to eliminate unimportant features according to the minimum redundancy and maximum relevancy (MRMR) criterion [39]. It was implemented with MIToolbox (Matlab version) [26]. As shown in Figure 19, the best average accuracy obtained is $99.76 \%$ with 60 features selected for designing the classifier in the $\mathrm{CV}$ experiment, while it achieves $96.90 \%$ accuracy for the classifier designed using 124 selected features in the ITT experiment.

\section{Discussion}

Cytology evaluation has been shown to be a safe, efficient and well-established technique for the diagnoses of many diseases. Its ability to reduce the mortality and morbidity of cervical cancer through mass screening is the most famous success. Classical cytological diagnosis is based on microscopic observation of specialized cells and

Table 4 Classification accuracies (\%) of different cell patterns

\begin{tabular}{ccccccccc}
\hline Exp. & Iter. & All & Diffused & Peripheral & Nucleolar & Coarse-speckled & Fine-speckled & Discr.-speckled \\
\hline 1 & - & 99.69 & 98.33 & 100 & 98.94 & 99.95 & 100 & 100 \\
\hline 2 & 1 & 97.65 & 93.98 & 99.24 & 82.98 & 98.88 & 85.71 & 99.33 \\
& 2 & 96.45 & 88.96 & 98.48 & 95.74 & 98.57 & 71.43 & 97.65 \\
& 3 & 97.23 & 93.65 & 97.72 & 87.23 & 98.47 & 78.57 & 99.66 \\
& 4 & 96.45 & 92.31 & 98.10 & 82.98 & 98.36 & 78.57 & 96.64 \\
& 5 & 97.13 & 94.98 & 96.96 & 89.36 & 98.47 & 71.43 & 98.66 \\
& 6 & 96.81 & 93.98 & 99.24 & 76.60 & 98.26 & 85.71 & 96.98 \\
& 7 & 96.81 & 94.31 & 97.34 & 87.23 & 98.67 & 82.14 & 95.64 \\
& 8 & 97.33 & 93.65 & 98.48 & 91.49 & 98.98 & 78.57 & 97.32 \\
& 9 & 96.50 & 90.30 & 98.48 & 93.62 & 98.47 & 85.71 & 95.97 \\
& 10 & 96.50 & 90.64 & 98.48 & 85.10 & 98.36 & 82.14 & 97.65 \\
\hline Avg & 96.87 & 92.68 & 98.25 & 87.23 & 98.55 & 80.00 & 97.55 \\
\hline
\end{tabular}

Classified accuracies of 6 patterns of cells and overall cells achieved for CV and ITT experiments. 


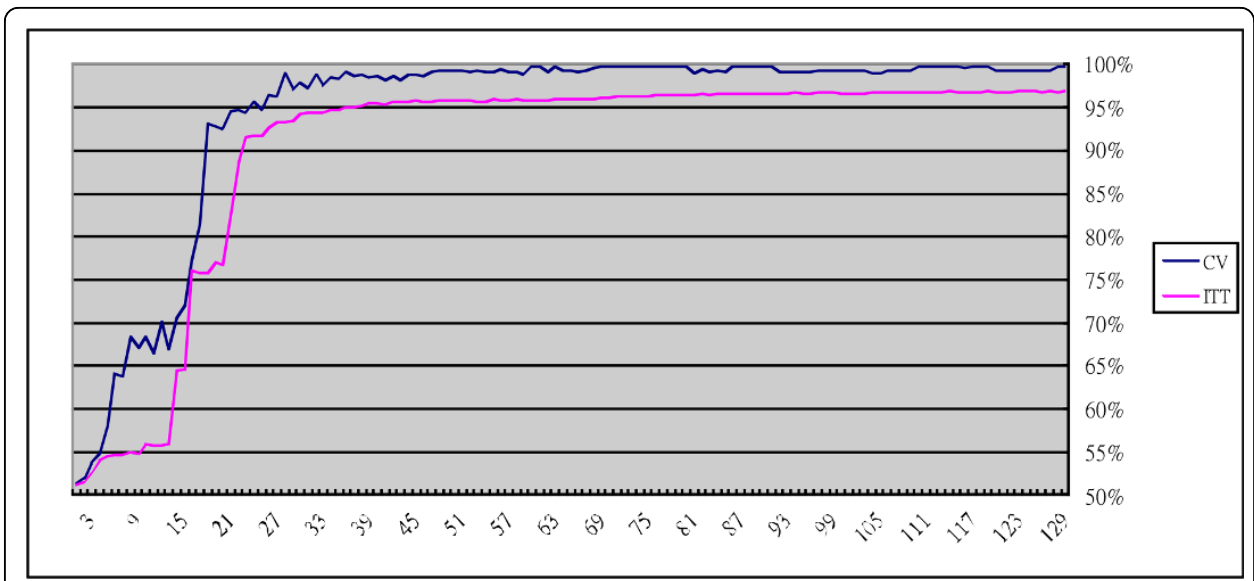

Figure 19 Comparison of accuracy against different number of features. Features selected using SVMRFE method compared between cross validation (CV) and independent training and testing (ITT) experiments.

qualitative assessment with descriptive criteria, which may result in inconsistent results because of subjective variability found in different observers [40]. Recently, automatic or semi-automatic computerized systems developed for segmenting and analyzing stained cervical cells from Pap smear images are demonstrated to be effective and efficient to assist pathologists in the diagnosis of abnormal cells [34,41-43] and in the discrimination of different types of cells $[34,44,45]$ through accurate and objective measurements of cell texture and morphology.

Tracing the cell migration, cell cycle, and cell differentiation from fluorescent microscopic images through automatic segmentation, classification, and tracking of living and cultured cells has also been widely conducted [46-48]. However, an automated image analysis system developed to fit a specific type, assay, or image set is hardly applicable to different cells acquired from different modalities [49]. Hence, techniques used for segmenting cells from visible-light microscopic images may not be directly applied in extracting cells from fluorescent microscopic images, whereas techniques used for extracting cells in a living cell population from fluorescent microscopic images may not be effective for processing IIF images.

Tested with the 3830 cells extracted from 196 images, the segmentation results show that PVO is greater than $89 \%$ and PVD is less than $22 \%$. The average classification accuracy achieved in this study is as high as $96.90 \%$ (error rate: $3.1 \%$ ) and $99.69 \%$ (error rate: $0.31 \%$ ) for CV and ITT experiments, respectively, which outperforms the performance reported in previous studies [3,5,6,16-19]. Table 5 compares the cell/image numbers and the error rates in classification of this study with other previous investigations.

Note that the cells included in the database used in the this study are quite different from the cells adopted in previous studies, which may induce bias when making comparisons. CellProfiler is a freely available software [49] useful for automatic cell segmentation as well as for quick and easy classification and scoring of cells with diverse cellular morphologies [48]. Figure 20 compares the segmented cell examples between the proposed method and the CellProfiler. It can be observed that the 
Table 5 Comparison of error rate between this study and previous investigations.

\begin{tabular}{cccc}
\hline Literature & Error rate & No. of cells/images & Validation method \\
\hline Perner et al. [6] & $23.60 \%$ & $\mathrm{NA} / 321$ & Human expert \\
& $25.00 \%$ & $\mathrm{NA} / 321$ & leave-one-out CV \\
Sack et al. [3] & $16.91 \%$ & $1041 / 676$ & $\mathrm{CV}$ \\
Elbischger et al. [17] & $9.75 \%$ & $982 / 38$ & IT (1:1) \\
Soda \& lannello [5] & $5.80 \%$ & $573 / 37$ & 8-fold CV \\
Rigon et al. [16] & $26.00 \%$ & $573 / 37$ & 8-fold CV \\
Huang et al. [18] & $7.60 \%$ & $1020 / \mathrm{NA}$ & 10-fold CV \\
Voigtet al. [19] & $6.00 \%$ & $\mathrm{NA} / 351$ & IT \\
This study & $0.31 \%$ & $3830 / 196$ & 5-fold CV \\
& $3.10 \%$ & $3830 / 196$ & ITT (1:1) \\
\hline
\end{tabular}

$\mathrm{CV}$ indicates cross validation and IT denotes independent training and testing.

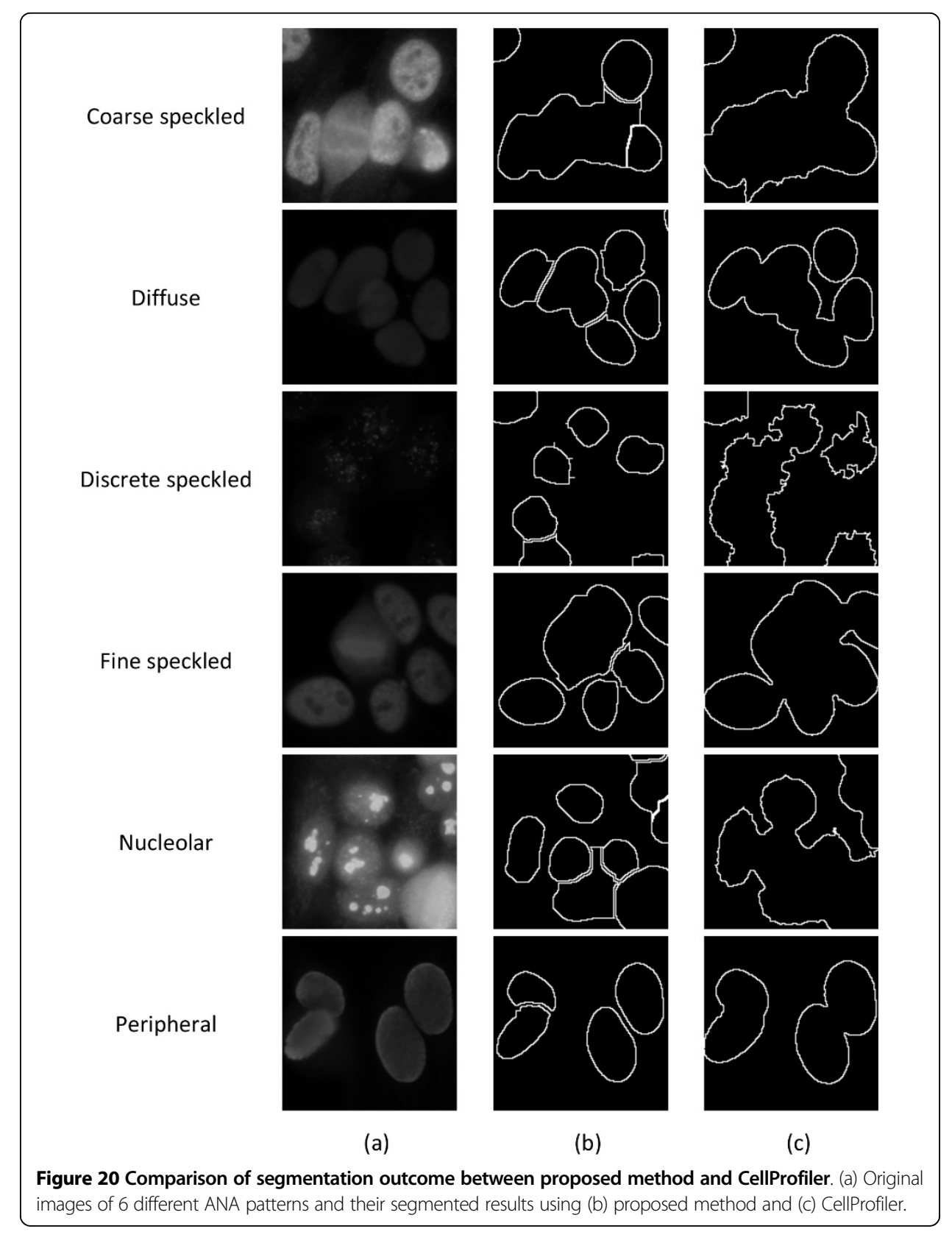


Table 6 Comparisons of number of segmented cells and classification performance between proposed method and CellProfiler evaluated based on PVO, PVD, RAE, and MHD.

\begin{tabular}{|c|c|c|c|c|c|c|c|c|c|c|c|}
\hline \multirow[t]{2}{*}{ Pattern } & \multicolumn{3}{|c|}{ Detected Cell Number } & \multicolumn{2}{|c|}{ PVO (\%) } & \multicolumn{2}{|c|}{ PVD (\%) } & \multicolumn{2}{|c|}{$\mathrm{RAE} \pm$ Std. } & \multicolumn{2}{|c|}{ MHD \pm Std. } \\
\hline & G. Truth & Proposed & CellProfiler & Proposed & CellProfiler & Proposed & CellProfiler & Proposed & CellProfiler & Proposed & CellProfiler \\
\hline Diffuse & 251 & 157 & 107 & 87.89 & 84.75 & 24.23 & 30.50 & $0.6386 \pm 0.42$ & $0.5248 \pm 0.36$ & $250.90 \pm 228.42$ & $510.69 \pm 250.56$ \\
\hline Peripheral & 191 & 153 & 97 & 93.47 & 72.43 & 13.06 & 55.15 & $0.3039 \pm 0.35$ & $0.5120 \pm 0.31$ & $87.15 \pm 182.86$ & $602.36 \pm 267.78$ \\
\hline Nucleolar & 285 & 175 & 101 & 91.94 & 84.90 & 16.12 & 30.20 & $0.4777 \pm 0.41$ & $0.6445 \pm 0.33$ & $157.99 \pm 208.70$ & $560.73 \pm 225.77$ \\
\hline Coarse speckled & 363 & 260 & 150 & 92.35 & 87.84 & 15.31 & 24.32 & $0.3506 \pm 0.36$ & $0.7038 \pm 0.21$ & $101.90 \pm 199.74$ & $556.02 \pm 261.51$ \\
\hline Fine speckled & 117 & 67 & 36 & 91.27 & 91.22 & 17.46 & 17.55 & $0.5423 \pm 0.41$ & $0.8637 \pm 0.13$ & $173.20 \pm 13.16$ & $458.46 \pm 21.41$ \\
\hline Discrete speckled & 216 & 195 & 125 & 78.03 & 69.64 & 43.95 & 60.71 & $0.5457 \pm 0.37$ & $0.7277 \pm 0.18$ & $143.99 \pm 202.59$ & $504.55 \pm 242.68$ \\
\hline
\end{tabular}


proposed method outperforms CellProfiler regarding the individual cells categorized into 6 different patterns.

In addition to PVO and PVD, other evaluation criteria, including, relative foreground area error (RAE) [50] and modified Hausdorff distance (MHD) [51], are also used to measure the segmentation errors. As can be seen in Table 6, the proposed method demonstrates better segmentation performance over the CellProfiler when evaluated based on PVO, PVD, RAE, and MHD. In addition, the number of miss-segment cells of the proposed method is less than the CellProfiler.

\section{Conclusion and future work}

In this study, a segmentation method was proposed to detect the boundaries of HEp-2 cells automatically, and then classification of cell patterns was performed based on the selected features. The results show that the proposed method can detect cells correctly in most image cases with PVO greater than $89 \%$ and PVD less than 22\%, whereas the best combination of selected features can achieve an average accuracy as high as $96.90 \%$ in discriminating 6 different types of cell patterns.

More cell images will be included in the dataset for verifying the segmentation performance and classification performance in the future. Furthermore, an automatic segmentation and classification system with graphical user interface (GUI) will be developed for computer-aid diagnosis. In fact, several different ANA patterns can appear in a single image, but the segmentation method proposed here only considers images with a unique cell pattern. Future works will focus on developing a segmentation method to extract cells with different patterns appearing in an image.

Competing interests

The authors declare that they have no competing interests.

\section{Authors' contributions}

CCC designed the software and conducted the image analysis; TYH recruited the patients, acquired the images, and verified the experimental results; CCC, JST, and YFC contributed to the discussion of the work and wrote the paper. All authors read and approved the final manuscript.

\section{Acknowledgements}

Funding for this article came from National Science Council of Taiwan under grant NSC100-2410-H-166-007-MY3. This article has been published as part of BioMedical Engineering OnLine Volume 12 Supplement 1, 2013: Selected articles from the 35th Annual International Conference of the IEEE Engineering in Medicine and Biology Society: Workshop on Current Challenging Image Analysis and Information Processing in Life Sciences. The full contents of the supplement are available online at http://www.biomedical-engineering-online.com/supplement/12/S1

\section{Authors' details}

${ }^{1}$ Department of Electrical Engineering, National Chung Hsing University, Taichung, Republic of China: Taiwan. ${ }^{2}$ Division of Allergy, Immunology and Rheumatology, Taichung Veterans General Hospital, Taichung, Republic of China: Taiwan. ${ }^{3}$ Department of Healthcare Administration, Central Taiwan University of Science and Technology, Taichung, Republic of China: Taiwan. ${ }^{4}$ Institute of Biomedical Engineering and Material Science, Central Taiwan University of Science and Technology, Taichung, Republic of China: Taiwan. ${ }^{5}$ Department of Health Services Administration, China Medical University, Taichung, Republic of China: Taiwan.

Published: 9 December 2013

References

1. Miller JF: Self-nonself discrimination and tolerance in T and B lymphocytes. Immunol Res 1993, 12(2):115-130.

2. Piazza A, Manoni F, Ghirardello A, Bassetti D, Villalta D, Pradella M, Rizzotti P: Variability between methods to determine ANA, antidsDNA and anti-ENA autoantibodies: A collaborative study with the biomedical industry. $J$ Immunol Methods 1998, 219:99-107.

3. Sack U, Knoechner S, Warschkau H, Pigla U, Emmerich MKF: Computer-assisted classification of HEp-2 immunofluorescence patterns in autoimmune diagnostics. Autoimmunity Reviews 2003, 2:298-304.

4. Rigon A, Soda P, Zennaro D, lannello G, Afeltra A: Indirect immunofluorescence (IIF) in autoimmune diseases: Assessment of digital images for diagnostic purpose. Cytometry Part B: Clinical Cytometry 2007, 72B:472-477. 
5. Soda P, lannello G: Aggregation of classifiers for staining pattern recognition in antinuclear autoantibodies analysis. IEEE T Inf Technol Biomed 2009, 13:322-329.

6. Perner P, Perner H, Müller B: Mining knowledge for Hep-2 cell image classification. J Artificial Intelligence in Medicine 2002, 26:161-173.

7. Huang YL, Chung CW, Hsieh TY, Jao YL: Adaptive automatic segmentation of HEp-2 cells in indirect immunofluorescence images. IEEE International Conference on Sensor Networks, Ubiquitous, and Trustworthy Computing 2008, 418-422.

8. Perner P: Image analysis and classification of HEp-2 cells in fluorescent images. Proceedings of the 14th International Conference on Pattern Recognition 1998, 2:1677.

9. Soda P, lannello G: A multi-expert system to classify fluorescent intensity in antinuclear autoantibodies testing. The 19th IEEE International Symposium on Computer-Based Medical Systems 2006, 219-224.

10. Soda P, lannello G: A Hybrid Multi-Expert Systems for HEp-2 Staining Pattern Classification. The 14th International Conference on Image Analysis and Processing 2007, 685-69.

11. Otsu N: Threshold selection method from gray-Llevel histograms. IEEE Transactions on Systems Man and Cybernetics 1979, 9:62-66.

12. Vincent $L$, Soille P: Watersheds in digital spaces: An efficient algorithm based on immersion simulations. IEEE Transactions on Pattern Analysis and Machine Intelligence 1991, 13(6):583-598.

13. Lotufo R, Falcao A: The ordered queue and the optimality of the watershed approaches. In Mathematical Morphology and its Application to Image and Signal Processing. Dordrecht: Kluwer Academic Publishers;Goutsias J, Vincent L, Bloomberg D 2000:341-345.

14. Creemers C, Guerti K, Geerts S, Cotthem KV, Ledda A, Spruyt V: HEp-2 cell pattern segmentation for the support of autoimmune disease diagnosis. Proceedings of the 4th International Symposium on Applied Sciences in Biomedical and Communication Technologies 2011, 28:Article 1-5.

15. Soda P, lannello G: Aggregation of classifiers for staining pattern recognition in antinuclear autoantibodies analysis. IEEE Transactions on Information Technology in Biomedicine 2009, 13:322-329.

16. Rigon A, Buzzulini F, Soda P, Onofri L, Arcarese L, lannello G, Afeltra A: Novel opportunities in automated classification of antinuclear antibodies on HEp-2 cells. Autoimmunity Reviews 2011, 10(10):647-652.

17. Elbischger P, Geerts S, Sander K, Ziervogel-Lukas GSinah P: Algorithmic framework for HEP-2 fluorescence pattern classification to aid auto-immune diseases diagnosis. IEEE International Symposium on Biomedical Imaging: From Nano to Macro 2009, 562-565.

18. Huang $Y C$, Hsieh TY, Chang CY, Cheng WT, Lin YC, Huang YL: HEp-2 cell images classification Bbased on textural and statistic features using self-organizing map. Lecture Notes in Computer Science 2012, 7197:529-538.

19. Voigt J, Krause C, Rohwäder E, Saschenbrecker S, Hahn M, Danckwardt M, Feirer C, Ens K, Fechner K, Barth E, Martinetz T, Stöcker W: Automated indirect immunofluorescence evaluation of antinuclear autoantibodies on HEp-2 cells. Clinical and Developmental Immunology 2012, 2012:1-7, (Article ID 651058).

20. Center for Disease Control: Quality assurance for the indirect immunofluorescence test for autoantibodies to nuclear antigen (IF-ANA): approved guideline. NCCLS I/LA2-A 1996, 16(11).

21. Solomon DH, Kavanaugh AJ, Schur PH: Evidence-based guidelines for the use of immunologic tests: Antinuclear antibody testing. Arthritis Care Res 2002, 47:434-444.

22. Soille P: Morphological image analysis: Principles and applications. New York: Springer-Verlag; 2003.

23. Collins D, Dai W, Peters T, Evens A: Automatic 3D model-based neuroanatomical segmentation. Human Brain Mapping 1995, 3(3):190-205.

24. Fischl B, Salat DH, Busa E, Albert M, Dieterich M, Haselgrove C, van der Kouwe A, Killiany R, Kennedy D, Klaveness S, Montillo A, Makris N, Rosen B, Dale AM: Whole brain segmentation: automated labeling of neuroanatomical structures in the human brain. Neuron 2002, 33:341-355.

25. Bae MH, Pan R, Wu T, Badea A: Automated segmentation of mouse brain images using extended MRF. Neuroimage 2009, 46(3):717-725.

26. Brown TT, Kuperman JM, Erhart M, White NS, Roddey JC, Shankaranarayanan A, Han ET, Rettmann D, Dale AM: Prospective motion correction of high-resolution magnetic resonance imaging data in children. Neuroimage 2010, 53(1):139-145.

27. Taur JS, Tao CW: Texture classification using a fuzzy texture spectrum and neural networks. Journal of Electronic Imaging 1998, 7(1):29-35.

28. Taur JS, Lee GH, Tao CW, Chen CC, and Yang CW: Segmentation of psoriasis vulgaris images using multiresolutionbased orthogonal subspace techniques. IEEE Trans System Man Cybern, Part-B 2006, 36(2):390-402.

29. Ojala T, Pietikainen M, Maenpaa T: Multiresolution gray-scale and rotation invariant texture classification with local binary patterns. IEEE Transactions on Pattern Analysis and Machine Intelligence 2002, 24(7):971-987.

30. Ahonen T, Hadid A, Pietikainen M: Face description with local binary patterns: Application to face recognition. IEEE Transactions on Pattern Analysis and Machine Intelligence 2006, 28(12):2037-2041.

31. Zhao G, Pietikainen M: Dynamic texture recognition using local binary patterns with an application to facial expressions. IEEE Transactions on Pattern Analysis and Machine Intelligence 2007, 29(6):915-928.

32. Vapnik VN: The nature of statistical learning theory New York: Springer-Verlag; 1995.

33. Chang CC, Lin CJ: Training v-support vector classifiers: Theory and algorithms. Neural Computation 2001, 13:2119-2147.

34. Chen YF, Huang PC, Lin KC, Lin HH, Wang LE, Cheng CC, Chen TP, Chan YK, Chiang JY: Semi-automatic segmentation and classification of Pap Ssmear cells. IEEE J Biomedical Health Informatics 2013.

35. Chang CC, Lin CJ: LIBSVM: a library for support vector machines 2001, Software available at http:/www.csie.ntu.edu.tw/ $\sim$ cjlin/libsvm.

36. Hsu CW, Chang CC, Lin CJ: A practical guide to support vector classification. 2003, Article available at http://www. csie.ntu.edu.tw/ cjlin/papers/guide/guide.pdf. Retrieved November 9, 2012.

37. Gutierrez-Osuna R: Introduction to Pattern Analysis, http://research.cs.tamu.edu/prism/lectures/pr/pr_l11.pdf, Retrieved November 9, 2012. 
38. Zhao YM, Yang ZX: Improving MSVM-RFE for multiclass gene selection. The Fourth International Conference on Computational Systems Biology 2010, 43-50.

39. Peng H, Long F, Ding C: Feature selection based on mutual information: Criteria of max-dependency, maxrelevance, and min-redundancy. IEEE Transactions on Pattern Analysis and Machine Intelligence 2005, 27:1226-1237.

40. DeMay RM: Common problems in Papanicolaou smear interpretation. Archives of Pathology \& Laboratory Medicine 1997, 121(3):229-238.

41. Plissiti ME, Nikou C, Charchanti A: Automated detection of cell nuclei in Pap smear images using morphological reconstruction and clustering. IEEE Trans Inf Technol Biomed 2011, 15(2):233-241.

42. Sulaimana SN, Isab NAM, Othmanc NH: Semi-automated pseudo colour features extraction technique for cervical cancer's pap smear images. Int J Knowledge-based Intell Eng Syst 2011, 15:131-143.

43. Bergmeir C, García-Silvente M, Benítez JM: Segmentation of cervical cell nuclei in high-resolution microscopic images: A new algorithm and a web-based software framework. Comput Methods Prog Biomed 2012, 107(3):497-512.

44. Sokouti B, Haghipour S, Tabrizi AD: A framework for diagnosing cervical cancer disease based on feedforward MLP neural network and ThinPrep histopathological cell image features. Neural Comput Appl 2012.

45. Gençtav A, Aksoy S, Önder S: Unsupervised segmentation and classification of cervical cell images. Pattern Recognition 2012, 45:4151-4168.

46. Chen X, Zhou X, Wong ST: Automated segmentation, classification, and tracking of cancer cell nuclei in time-lapse microscopy. IEEE Trans Biomed Eng 2006, 53(4):762-766

47. Du TH, Puah WC, Wasser M: Cell cycle phase classification in 3D in vivo microscopy of Drosophila embryogenesis. BMC Bioinformatics 2011, 12(s13):1-9.

48. Jones TR, Carpenter AE, Lamprecht MR, Moffat J, Silver SJ, Grenier JK, Castoreno AB, Eggert US, Root DE, Golland P, Sabatini DM: Scoring diverse cellular morphologies in image-based screens with iterative feedback and machine learning. Proc Natl Acad Sci 2009, 106(6):1826-1831.

49. Carpenter AE, Jones TR, Lamprecht MR, Clarke C, Kang IH, Friman O, Guertin DA, Chang JH, Lindquist RA, Moffat J, Golland P, Sabatini DM: CellProfiler: image analysis software for identifying and quantifying cell phenotypes. Genome Biology 2006, 7(10):R100.

50. Sahoo PK, Soltani S, Wong AK, Chan YC: A survey of thresholding techniques. Computer Vision Graphics Image Processing 1988, 41(2):233-260.

51. Sezgin M, Sanlur B: Survey over image thresholding techniques and quantitative performance evaluation. Journal of Electronic Imaging 2004, 13(1):146-165.

doi:10.1186/1475-925X-12-S1-S5

Cite this article as: Cheng et al: An automatic segmentation and classification framework for anti-nuclear antibody images. BioMedical Engineering OnLine 2013 12(Suppl 1):S5.

\section{Submit your next manuscript to BioMed Central and take full advantage of:}

- Convenient online submission

- Thorough peer review

- No space constraints or color figure charges

- Immediate publication on acceptance

- Inclusion in PubMed, CAS, Scopus and Google Scholar

- Research which is freely available for redistribution 